\title{
Amplification and generation of terahertz plasmons in gated two-dimensional channels: Modal analysis
}

\author{
O. Sydoruk ${ }^{\mathrm{a})}$ \\ Optical and Semiconductor Devices Group, Department of Electrical and Electronic Engineering, \\ Imperial College London, Exhibition Road, London SW7 2AZ, United Kingdom
}

(Received 14 April 2014; accepted 12 May 2014; published online 27 May 2014)

\begin{abstract}
A theoretical analysis is presented of plasmons in gated two-dimensional electron channels supporting dc currents. In contrast to previous treatments, the model takes into account complete mode spectra and transverse field distributions. Conditions for plasmon amplification are determined, and asymmetric plasmonic oscillators are analysed. The results are compared with the traditional treatment of the Dyakonov-Shur instability. The limitations of the traditional treatment are thus revealed. The present electrodynamically rigorous model can be used to design and analyse terahertz plasmonic oscillators. (C) 2014 AIP Publishing LLC. [http://dx.doi.org/10.1063/1.4879317]
\end{abstract}

\section{INTRODUCTION}

The pioneering works of Dyanokov and Shur on terahertz $(\mathrm{THz})$ generation ${ }^{1}$ and detection ${ }^{2}$ have rekindled interest in plasmons in two-dimensional electron channels. The proposal for $\mathrm{THz}$ sources is particularly interesting: highpower and efficient $\mathrm{THz}$ generation has been notoriously difficult to achieve, and the existing sources are bulky and expensive. The idea of Dyakonov and Shur ${ }^{1}$ relies on an instability of plasmons propagating in a field-effect transistor (FET) when a dc current flows through the channel. Plasmon instabilities had been considered before, but mostly relying on the travelling-wave interactions where plasmons grow while propagating along the device, see for example Refs. 3-5. In contrast, plasmons in the Dyakonov-Shur model (reminiscent of the Pierce instability ${ }^{6}$ ) grow when they reflect from the contacts.

Clearly, the properties of plasmon reflection determine whether the instability can occur. To describe the reflection theoretically, Dyakonov and Shur ${ }^{1}$ made several assumptions. First, they considered the boundary conditions at a single point on the channel, ignoring the rest of the contact. Second, because they found that symmetric boundary conditions lead to no oscillations, they postulated asymmetric boundary conditions, zero ac potential at the source and zero ac current at the drain. This elegant and simple approach has dominated the literature (see, for example, Refs. 7-11).

However, more rigorous models have emerged in recent years. Solving Maxwell's equations and using the Fourier analysis and, Popov et al. ${ }^{12}$ studied plasmon generation and coupling to electromagnetic radiation in one-dimensional FET arrays. Sydoruk et al. ${ }^{13,14}$ developed a semi-analytical model based on mode matching. The latter, in contrast to the traditional treatment of Dyakonov and Shur, relies on electrodynamic boundary conditions and takes into account the field distributions along an entire contact. It has been used to predict distributed gain in channels with multiple sections, ${ }^{13}$

${ }^{\text {a)} E l e c t r o n i c ~ m a i l: ~ o s y d o r u k @ i m p e r i a l . a c . u k ~}$ to study plasmon amplification at contacts, ${ }^{14,15}$ and to consider effects of electron collisions and diffusion. ${ }^{16}$

This paper develops further the mode-matching technique for plasmon amplification. It considers gated electron channels: closed by a conductor on one side of the channel but open on the other side. In contrast, previous papers considered either open channels ${ }^{15,16}$ or channels closed on both sides. $^{13,14}$ Section II introduces the model. Section III considers reflection from a conducting boundary (a source or a drain) and demonstrates plasmon amplification. Section IV compares the mode-matching technique with the traditional model of Dyakonov and Shur. Section V draws conclusions.

\section{CONFIGURATION AND MODE SPECTRUM}

This section discusses the theoretical model and considers the mode spectrum. The configuration consists of a twodimensional electron channel placed at $x=0$, see Fig. 1 . The channel is surrounded by a dielectric with the relative permittivity $\varepsilon_{\mathrm{d}}$. A perfectly conducting gate is placed above the channel, at $x=d$. Below the channel, the dielectric extends to $x=-\infty$.

Electrons drift in the channel along the $z$-direction with the dc velocity $v_{0}$. They will interact with an electromagnetic wave if the wave has an electric field component along the channel. Therefore, TM waves will be assumed with the electric field components $E_{z}$ and $E_{x}$ and the magnetic field component $H_{y}$. They obey Maxwell's equations in the dielectric above and below the channel, and their amplitudes have the form

$$
\begin{aligned}
& E_{x}^{(1,2)}=A^{(1,2)} \mathrm{e}^{-\mathrm{j} k_{x} x}+B^{(1,2)} \mathrm{e}^{\mathrm{j} k_{x} x} \\
& H_{y}^{(1,2)}=\frac{\omega \varepsilon_{0} \varepsilon_{\mathrm{d}}}{k_{z}} E_{x}^{(1,2)} \\
& E_{z}^{(1,2)}=-\frac{k_{x}}{k_{z}}\left(A^{(1,2)} \mathrm{e}^{-\mathrm{j} k_{x} x}-B^{(1,2)} \mathrm{e}^{\mathrm{j} k_{x} x}\right) .
\end{aligned}
$$

Here, $A^{(1,2)}$ and $B^{(1,2)}$ are constants, and the superscripts (1) and (2) refer to the space above and below the channel, respectively. The longitudinal wavenumber is $k_{z}$; the transverse one is $k_{x}$; the angular frequency is $\omega$; the vacuum 


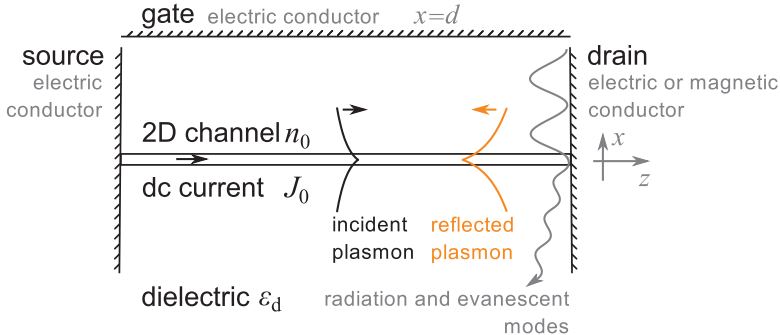

FIG. 1. The plasmons propagating along and against the dc current in gated electron channels have different wavenumbers and field distributions (shown schematically in orange and black). Plasmon reflection from a contact will, therefore, excite not only a reflected plasmon but also a continuum of radiation modes (shown schematically in grey). For comparison with the traditional model of Dyakonov and Shur, ${ }^{1}$ the contacts can be either electric or magnetic conductors.

permittivity is $\varepsilon_{0}$. The wavenumbers obey the dielectric dispersion relation is the form

$$
k_{x}^{2}+k_{z}^{2}=\varepsilon_{\mathrm{d}} \frac{\omega^{2}}{c^{2}},
$$

where $c$ is the light velocity in vacuum.

The fields obey the standard boundary conditions. At the gate:

$$
\left.E_{z}^{(1)}\right|_{x=d}=0
$$

and at the channel:

$$
\begin{aligned}
\left.E_{z}^{(1)}\right|_{x=0} & =\left.E_{z}^{(2)}\right|_{x=0} \\
\left.E_{x}^{(1)}\right|_{x=0}-\left.E_{x}^{(2)}\right|_{x=0} & =\frac{e n}{\varepsilon_{0} \varepsilon_{\mathrm{d}}} \\
\left.H_{y}^{(1)}\right|_{x=0}-\left.H_{y}^{(2)}\right|_{x=0} & =J .
\end{aligned}
$$

Here, $e$ is the electron charge, and $n$ and $J$ are the amplitudes of the ac electron charge and current density, respectively. As is usually done, the ac quantities are assumed to be much smaller than their dc counterparts: $|n| \ll n_{0},|v| \ll v_{0}$, where $n_{0}$ is the dc electron density and $v$ is the amplitude of the ac electron velocity. The current density can then be linearized leading to the expression $J=e n_{0} v+e n v_{0}$. The amplitude of the ac velocity can be found from the linearized Newton equation of motion as

$$
v=-\mathrm{j} \frac{e}{m} \frac{\left.E_{z}\right|_{x=0}}{\omega-k_{z} v_{0}},
$$

where $m$ is the electron effective mass. Substituting the expressions for the fields Eq. (1) into the boundary conditions, Eqs. (3) and (4) give for the amplitude of the $E_{x}$ field below the channel

$$
\begin{aligned}
E_{x}^{(2)}= & A\left[\left(1+\Gamma-\Gamma \mathrm{e}^{-2 \mathrm{j} k_{x} d}\right) \mathrm{e}^{-\mathrm{j} k_{x} x}\right. \\
& \left.+\left(\Gamma+(1-\Gamma) \mathrm{e}^{-2 \mathrm{j} k_{x} d}\right) \mathrm{e}^{\mathrm{j} k_{x} x}\right],
\end{aligned}
$$

where $A$ is a constant and

$$
\Gamma=-\mathrm{j} \frac{\Omega_{\mathrm{p}}^{2} k_{x}}{\left(\omega-k_{z} v_{0}\right)^{2}}
$$

with $\Omega_{\mathrm{p}}^{2}=e^{2} n_{0} /\left(2 \varepsilon_{0} \varepsilon_{\mathrm{d}} m\right)$.
In principle, the transverse wavenumber may be complex, $k_{x}=k_{x}^{\prime}+\mathrm{j} k_{x}^{\prime \prime}$. However, the fields cannot grow at $x=-\infty$ (although they may remain finite). ${ }^{17,18}$ This condition restricts the permissible values of $k_{x}$, allowing the eigenmode spectrum to be separated into two parts, plasmons and radiation modes, as discussed next.

\section{A. Plasmons}

If the transverse wavenumber $k_{x}$ has a non-negative imaginary part, $k_{x}^{\prime \prime}>0$, the term in front of $\exp \left(\mathrm{j} k_{x} x\right)$ in Eq. (6) must vanish for the fields to remain finite, so that

$$
\Gamma+(1-\Gamma) \mathrm{e}^{-2 \mathrm{j} k_{x} d}=0
$$

or, rearranging,

$$
-\mathrm{j} \frac{e^{2} n_{0}}{\varepsilon_{0} \varepsilon_{\mathrm{d}} m} \frac{k_{x}}{\left(\omega-k_{z} v_{0}\right)^{2}}=1+\mathrm{j} \cot \left(k_{x} d\right) .
$$

Equation (8) is the dispersion relation for plasmons in gated channels, and its solution gives imaginary values of $k_{x}$ and real values of $k_{z}$, so that the fields decay away from the channel. Ignoring retardation, $k_{x}=\mathrm{j} k_{z}$, Eq. (8) reduces to the expressions given by Theis ${ }^{19}$ and Tsui et al. ${ }^{20}$ in the absence and by Crowne ${ }^{7}$ in the presence of drift.

So far, most attention in the literature has been paid to two limiting cases: fully gated and open channels. For fully gated channels, $k_{z} d \rightarrow 0$. It has also been referred to as the shallow-water limit by Dyakonov and Shur. ${ }^{1}$ The dispersion relation reduces then to

$$
\omega-k_{z} v_{0}= \pm s k_{z},
$$

where $s$ is the phase velocity of the plasmons, which for the fully gated channels is of the form $s=\sqrt{e^{2} n_{0} d /\left(\varepsilon_{0} \varepsilon_{\mathrm{d}} m\right)}$.

Open (or ungated) channels correspond to the opposite limit $k_{z} d \rightarrow \infty$, also referred to as the deep-water limit. ${ }^{10}$ The dispersion relation is then of the form ${ }^{7,10,15,21}$

$$
\omega-k_{z} v_{0}= \pm \Omega_{\mathrm{p}} \sqrt{k_{z}} \text {. }
$$

Both Eqs. (10) and (11) have been derived ignoring retardation.

Solutions of the dispersion relations Eqs. (9)-(11) are different for the opposite directions, i.e., for the opposite values of $k_{z}$. It means that counter-propagating plasmons may have different phase and group velocities and, as will be shown later, different field distributions.

Up two four plasmons described by Eqs. (9) and (11) can propagate at the same frequency, two with low and two with high wavenumbers. Some studies have indeed taken all four of them into account. ${ }^{7,14}$ However, as numerical calculations show, ${ }^{14}$ amplification upon reflection can be explained solely by an interplay of the low-wavenumber plasmons. Figures 2(a)-2(c) show their dispersion curves for three values of the gate-to-channel distance $d: 25,75$, and $250 \mathrm{~nm}$. All three figures were calculated for GaAs channels $\left(\varepsilon_{\mathrm{d}}=12.8, \quad m=0.067 m_{0}\right) \quad$ with the electron density $n_{0}=10^{11} \mathrm{~cm}^{-2}$. The electron drift velocity was 


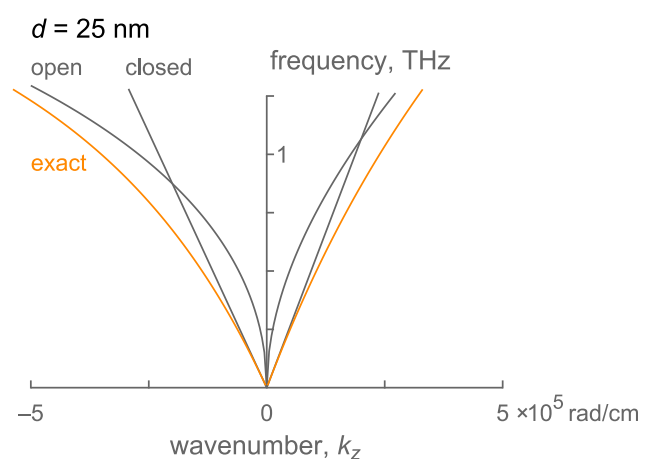

(a)

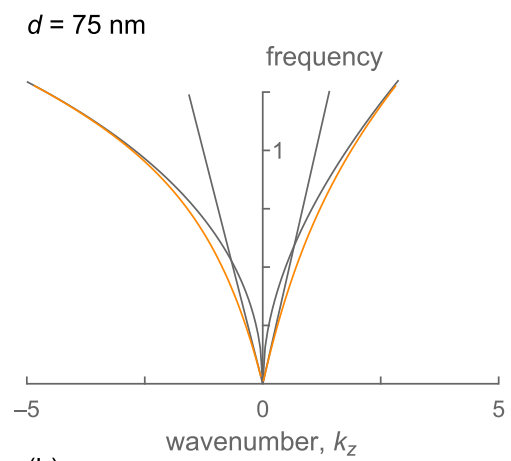

(b)

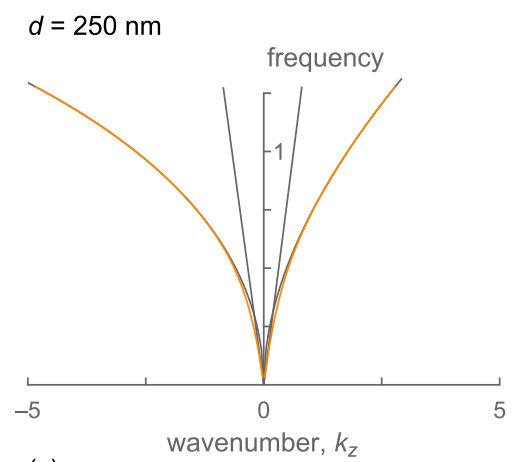

(c)

FIG. 2. In the presence of dc current, the plasmon dispersion curves are asymmetric. Depending on the gate-to-channel distance and on the frequency, the exact solution (orange lines) can be approximated by the solutions either for fully closed or open channels (grey lines).

$v_{0}=3 \times 10^{6} \mathrm{~cm} / \mathrm{s}$. It is ten times smaller than the maximum drift velocity achievable in GaAs and corresponds to the dc current density of about $0.05 \mathrm{~A} / \mathrm{cm}$.

The dispersion curves are asymmetric: at the same frequency, the plasmons propagating against the dc current (negative $k_{z}$ ) have higher wavenumbers than the plasmons propagating along the current. Whether a solution of the general dispersion relation Eq. (9) can be approximated either by the solution for an open, Eq. (10), or a fully closed channel, Eq. (11), depends not only on the gate-to-channel distance but also on the frequency. So, for $d=25 \mathrm{~nm}$, the exact solution (orange lines) follows the fully closed-channel solution (straight gray lines) for frequencies below $0.25 \mathrm{THz}$, see Fig. 2(a). Between 0.25 and $1.25 \mathrm{THz}$, the exact solution deviates from both approximations. On the other hand, for $d=75 \mathrm{~nm}$, the exact solution follows the fully closed- channel one up to $0.25 \mathrm{THz}$. It follows the open-channel solution for frequencies above $1 \mathrm{THz}$, see Fig. 2(b). For $d=250 \mathrm{~nm}$, the exact and the open-channel solutions coincide except for very low frequencies, see Fig. 2(c).

Once the wavenumbers are known, the plasmonic fields can be calculated. So, the $E_{x}$ components above and below the channel are of the form

$$
\begin{aligned}
& E_{x}^{(1)}=A \frac{\cos \left[k_{x}(x-d)\right]}{\sin \left(k_{x} d\right)} \\
& E_{x}^{(2)}=\mathrm{j} A \mathrm{e}^{-\mathrm{j} k_{x} x} .
\end{aligned}
$$

The corresponding current density and the electron velocity are

$$
\begin{aligned}
& J=A \frac{\omega \varepsilon_{0} \varepsilon_{\mathrm{d}}}{k_{z}} \frac{\mathrm{e}^{-\mathrm{j} k_{x} d}}{\sin \left(k_{x} d\right)} \\
& v=-\frac{e}{m} \frac{k_{x}}{k_{z}\left(\omega-k_{z} v_{0}\right)} .
\end{aligned}
$$

The fields of the counter-propagating plasmons will be different due to the different plasmon wavenumbers $k_{x}$ and $k_{z}$. Figure 3 compares the magnitude of the $E_{x}$ component of the counter-propagating plasmons. The frequency is $0.25 \mathrm{THz}$ and the other parameters are the same as in Fig. 2. For comparison of the decay rates, the fields above and below the channel were normalized separately.

For $d=25 \mathrm{~nm}$, the fields resemble most closely those expected in fully closed channels. In particular, the fields for the both plasmons are almost constant and coincide above the channel, see Fig. 3(a). Nevertheless, the fields decay differently below the channel. For $d=75 \mathrm{~nm}$, the fields above the channel decay before reaching the gate and they are somewhat different for the counter-propagating plasmons, see Fig. 3(b). For $d=250 \mathrm{~nm}$, the plasmons have different distributions above and below the channel, see Fig. 3(c).

The ac power carried by the plasmons is the sum of the electromagnetic power due to the fields and the kinetic power due to the electron motion. ${ }^{14,15,22,23}$ It can be written in the form

$$
\begin{aligned}
P= & \frac{1}{2} \operatorname{Re} \int_{-\infty}^{d} E_{x} H_{y}^{*} d x+\frac{m v_{0}}{2 e} \operatorname{Re}\left(v J^{*}\right) \\
= & |A|^{2} \frac{\omega \varepsilon_{0} \varepsilon_{\mathrm{d}}}{k_{z} k_{x}^{\prime \prime} \sinh ^{2}\left(k_{x}^{\prime \prime} d\right)}\left[k_{x}^{\prime \prime} d+\sinh \left(k_{x}^{\prime \prime} d\right) \mathrm{e}^{k_{x}^{\prime \prime} d}\right. \\
& \left.\times\left(1+\frac{2 k_{x}^{\prime \prime 2} v_{0}}{k_{z}\left(\omega-k_{z} v_{0}\right)}\right)\right] .
\end{aligned}
$$

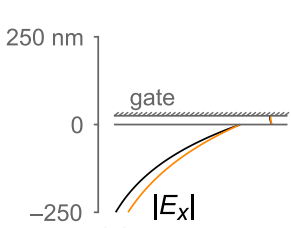

(a)

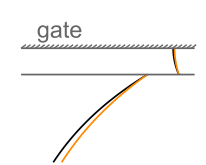

(b)

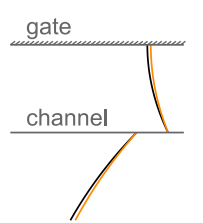

(c)
FIG. 3. Counter propagating plasmons have different transverse field distributions (black and orange lines) for all values of the gate-to-channel distance. This difference causes mismatched plasmonic fields at boundaries and complicates plasmon reflection. 


\section{B. Radiation modes}

If the transverse wavenumber, $k_{x}$, is an arbitrary real number, all of the terms in Eq. (6) remain finite at $x=-\infty$. These radiation modes form a continuous spectrum with the amplitudes of the $E_{x}$ fields above and below the channel of the form

$$
\begin{aligned}
& e_{x}^{(1)}=A \frac{\cos \left[k_{x}(x-d)\right]}{\sin \left(k_{x} d\right)} \\
& e_{x}^{(2)}=A\left(\frac{\cos \left[k_{x}(x-d)\right]}{\sin \left(k_{x} d\right)}+2 \mathrm{j} \Gamma \cos k_{x} x\right) .
\end{aligned}
$$

The corresponding amplitudes of the ac current density and velocity are

$$
\begin{aligned}
J & =-2 \mathrm{j} A \frac{\omega \varepsilon_{0} \varepsilon_{\mathrm{d}}}{k_{z}} \Gamma \tan k_{x} d \\
v & =-A \frac{e}{m} \frac{k_{x}}{k_{z}} \frac{\tan k_{x} d}{\omega-k_{z} v_{0}} .
\end{aligned}
$$

The following section uses the expressions for the ac fields, Eqs. (12) and (15), to solve the problem of plasmon reflection. The expressions for the amplitude of the ac current and velocity, Eqs. (13) and (16), are needed to calculate the ac power, see Eq. (14).

\section{REFLECTION FROM A CONDUCTING BOUNDARY}

When a plasmon reaches a contact, it reflects and propagates in the opposite direction. Assuming a perfectly conducting electric contact at $z=0$, the boundary condition is the absence of the transverse electric field, $\left.E_{x}\right|_{z=0}=0$. This condition should hold everywhere at the contact, $-\infty<x \leq d$. If no dc current flows, the incident and the reflected plasmons have the same transverse distributions and, hence, the same amplitudes.

However, they have different field distributions in the presence of a dc current, see Fig. 3. Therefore, first, the incident and reflected plasmon can have different amplitudes and, second, the radiation modes will be excited at the contact to satisfy the boundary condition. ${ }^{13-16}$ So, the boundary condition takes the form

$$
E_{x}^{(\mathrm{i})}+R E_{x}^{(\mathrm{r})}+\int_{0}^{\infty} r\left(k_{x}\right) e_{x}^{(\mathrm{r})} \mathrm{d} k_{x}=0 .
$$

Here, the superscripts (i) and (r) correspond, respectively, to the incident and reflected waves. The amplitude of the incident plasmon is unity, so $R$ is the (amplitude) plasmon reflection coefficient; $r\left(k_{x}\right)$ is the amplitude of the radiation modes.

Two methods to solve Eq. (17), one analytical and one numerical, were previously considered in Refs. 13-16. The analytical method assumes that the dc current is weak and causes perturbations in the ac quantities that are linear with the drift velocity. Relying on orthogonality of the driftless modes, an approximate expression for the plasmon reflection coefficient can be derived as (see Appendix A)

$$
R=-1+\frac{4 k_{x}^{(0)} v_{0}}{\omega} \times \frac{\Gamma^{(0)} \sin ^{2}\left(k_{x}^{(0)} d\right)\left[k_{x}^{(0)} d-2 \mathrm{j} \Gamma^{(0)} \sin ^{2}\left(k_{x}^{(0)} d\right)+2\left(k_{x}^{(0)} d\right)^{2} \cot \left(k_{x}^{(0)} d\right)\right]}{\left[k_{x}^{(0)} d-2 \mathrm{j} \Gamma^{(0)} \sin ^{2}\left(k_{x}^{(0)} d\right)\right]^{2}},
$$

where the superscript (0) denotes driftless quantities and $k_{x}^{(0)} \approx \mathrm{j} k_{z}^{(0)}$.

This expression simplifies greatly for open $\left(k_{z} d \rightarrow \infty\right)$ and fully closed $\left(k_{z} d \rightarrow 0\right)$ channels. In both limits, the reflection coefficient is

$$
R=-1-2 \frac{v_{0}}{s^{(0)}},
$$

where $s^{(0)}$ is the phase velocity of the driftless plasmon, which is different for open and closed channels.

The second, numerical, method to solve Eq. (17) is based on multiplying it consequently by $E_{x}^{(\mathrm{i})}, E_{x}^{(\mathrm{r})}$, and $e_{x}^{(\mathrm{r})}$ and integrating the expressions over $x$ (see Appendix B). The resulting equations depend only on $k_{x}$ and can be solved numerically, for example, by presenting them in a truncated matrix form or by the method of iterations.

Figure 4 shows the amplitudes of the reflection coefficient, $|R|$, depending on the dc current density. A current density is positive when the plasmon is incident on the boundary along the dc current. It is negative when the plasmon is incident in the direction opposite to the dc current. The results are for the channel with the same parameters as above; for three values of the gate-to-channel distance $d$ and at three values of the frequency.

Figure 4(a) shows results calculated both numerically (orange line) and the analytically (black line). The results agree. The same agreement was observed for the parameters of all other Fig. 4, so they show only the numerical curves.

Figure 4 reveals the following general trends. First, the effect of the dc current (how much $|R|$ deviates from unity) increases with the dc current density. Second, the effect is larger for higher frequencies. Third, at the same frequency, it is larger for smaller gate-to-channel distances. These trends can be explained by looking at the approximate expression for the reflection coefficient, Eq. (19), and at the dispersion diagrams of Fig. 2. At a fixed value of $v_{0}$, the dc current affects stronger the reflection coefficient of the plasmons with lower phase-velocities. As Fig. 2 shows, the phase velocities increase with the frequency, and they are the largest for the smallest value of $d$. 


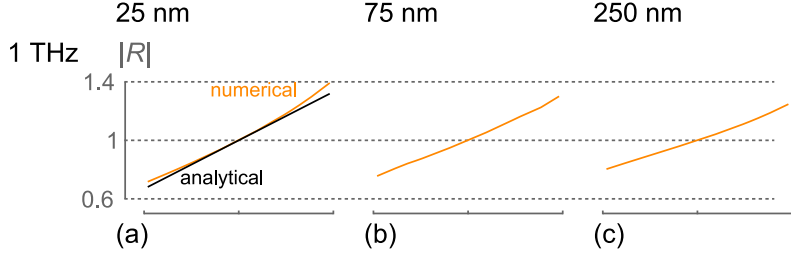

(a)

(b)

(c)

$0.5 \mathrm{THz}$

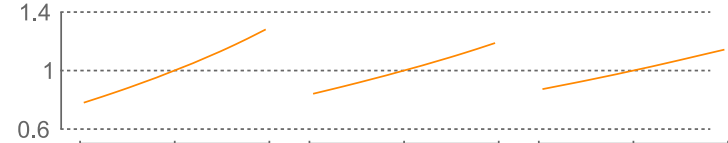

(d)

(e)

(f)

$0.25 \mathrm{THz}$

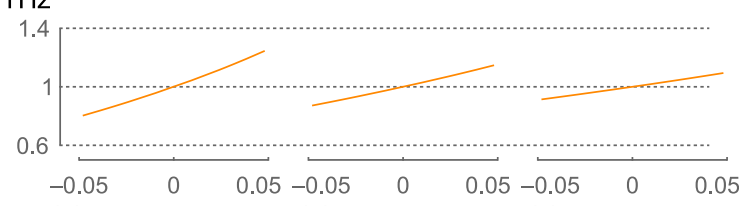

(g)

(h)

(k)

dc current density, $\mathrm{A} / \mathrm{cm}$

FIG. 4. The effect of the dc current on the amplitude reflection coefficient $|R|$ is larger for higher frequencies and lower gate-to-channel distances. Numerical [orange lines] and analytical [black line in (a)] results agree. The effects for the opposite values of the dc current cancel each other, which implies that symmetric resonators do not oscillate.

Comparing pairs of the results reveals further trends. In the limit of fully closed channels, the phase velocity is constant with frequency, and so should be the effect of the dc current on the reflection coefficient. As, for example, Fig. 2(a) shows, this limit is applicable for $d=25 \mathrm{~nm}$ at low frequencies. And indeed, the curves in the corresponding Figs. 4(d) and 4(g) are similar to each other.

Also, for fully closed channels, the effect of the current increases as the square root of the gate-to-channel distance, because $s^{(0)} \sim \sqrt{d}$. It is confirmed by Figs. 4(g) and 4(h), for $d=25$ and $75 \mathrm{~nm}$. The maximum values of $|R|$ are 1.25 and 1.14 , so that $0.14 / 0.25=1.79 \approx 1.73=\sqrt{75 / 25}$, in agreement with Eq. (19).

At $1 \mathrm{THz}$, the dispersion curves for $d=75$ and $250 \mathrm{~nm}$ are almost identical [see Figs. 2(b) and 2(c)], and so are the corresponding curves in Figs. 4(b) and 4(c). On the other hand, for fully open channels, the effect of the current should increase linearly with frequency, since $s^{(0)} \sim \omega^{-1}$. And indeed, for Figs. 4(c) and 4(f), the maximum values of $|R|$ are 1.25 and 1.14 , so that $0.25 / 0.14 \approx 2$ which is the ratio of the frequencies in these figures.

A resonator made from a homogeneous channel and two electric conductors as mirrors will have the roundtrip gain of $G=\left.\left.R\right|_{v_{0}} \cdot R\right|_{-v_{0}}$. The plasmons are incident along the dc current (positive $v_{0}$ ) for one conductor and against the dc current (negative $v_{0}$ ) for the other. For all of Fig. $4,\left.\left.R\right|_{v_{0}} \cdot R\right|_{-v_{0}}=1$. Such a symmetric resonator does not oscillate in the presence of a dc current.

Although the amplitude reflection coefficients can characterize resonators with multiple reflectors, they do not describe completely the effects of single reflectors. To show whether a single reflector can amplify plasmons, the power reflection coefficient should be used, defined as

$$
R_{\mathrm{p}}=|R|^{2} \frac{P^{(\mathrm{i})}}{P^{(\mathrm{r})}},
$$

where $P^{(\mathrm{i})}$ and $P^{(\mathrm{r})}$ are the power of the incident and the reflected plasmons, Eq. (14) with $|A|=1$. Figure 5 shows the power reflection coefficient for $d=25 \mathrm{~nm}$ at $1 \mathrm{THz}$, corresponding to Fig. 4(a). The plasmons are amplified for negative dc currents (flowing away from the conductor) and deamplified for positive currents. The behavior is the same as for open channels. ${ }^{15}$

\section{PLASMONIC OSCILLATORS AND THE DYAKONOV-SHUR INSTABILITY}

The above result that symmetric resonators do not oscillate agrees with the original conclusion by Dyakonov and Shur. ${ }^{1}$ To circumvent the problem, they postulated a resonator with asymmetric boundary conditions: zero ac potential at the source and zero ac current at the drain. The electrodynamic analogues are the perfect electric conductor for the source and the perfect magnetic conductor for the drain, see Fig. 1. The boundary condition for the magnetic conductor is $H_{y}=0$ and it can be written, analogously to Eq. (17), in the form

$$
H_{y}^{(\mathrm{i})}+R_{\mathrm{mag}} H_{y}^{(\mathrm{r})}+\int_{0}^{\infty} r_{\mathrm{mag}}\left(k_{x}\right) h_{y}^{(\mathrm{r})} \mathrm{d} k_{x}=0 .
$$

For TM waves, however, $H_{y}=\left(\omega \varepsilon_{0} \varepsilon_{\mathrm{d}} / k_{z}\right) E_{x}$, and Eq. (21) can be rewritten as

$$
E_{x}^{(\mathrm{i})}+R_{\operatorname{mag}} \frac{k_{z}^{(\mathrm{i})}}{k_{z}^{(\mathrm{r})}} E_{x}^{(\mathrm{r})}+\int_{0}^{\infty} r_{\mathrm{mag}}\left(k_{x}\right) \frac{k_{z}^{(\mathrm{i})}}{k_{z}} e_{x}^{(\mathrm{r})} \mathrm{d} k_{x}=0 .
$$

Comparing Eq. (22) to Eq. (17) shows that once the reflection coefficient $R$ for the electric conductor is known, the reflection coefficient for the magnetic conductor can be found, without solving Eq. (21), as

$$
R_{\text {mag }}=\frac{k_{z}^{(\mathrm{r})}}{k_{z}^{(\mathrm{i})}} R \text {. }
$$

The roundtrip gain of the resonator from Fig. 1 is then

$$
G=\left.\left.R\right|_{v_{0}} \cdot R_{\mathrm{mag}}\right|_{-v_{0}}=\left.\left.\frac{\left.k_{z}\right|_{v_{0}}}{\left.k_{z}\right|_{-v_{0}}} R\right|_{v_{0}} \cdot R\right|_{-v_{0}}=\frac{\left.k_{z}\right|_{v_{0}}}{\left.k_{z}\right|_{-v_{0}}}
$$

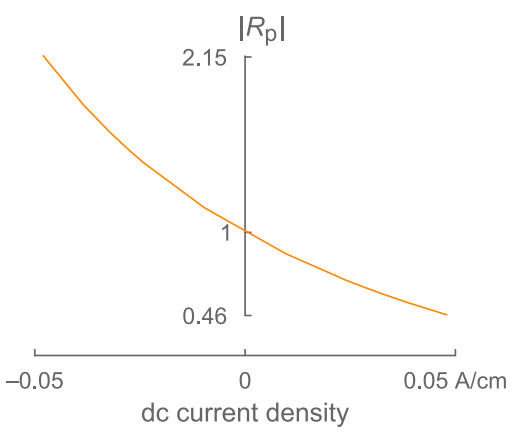

FIG. 5. The power reflection coefficient shows that plasmons can amplify or deamplify when reflecting from an electric boundary. They amplify when incident on the boundary against the dc current (negative current density). 
because $\left.\left.R\right|_{v_{0}} \cdot R\right|_{-v_{0}}=1$. In particular, for fully closed channels, $G=\left(s+v_{0}\right) /\left(s-v_{0}\right)$. The original treatment by Dyakonov and Shur ${ }^{1}$ as well as many that followed ${ }^{7,8,10}$ considered the boundary conditions only at the channel (i.e., only at $x=0$ ) and ignored the transverse field distributions. They also ignored the radiation modes. Under these assumptions, the boundary conditions Eq. (17) and Eq. (21) become simply

$$
\begin{aligned}
1+R & =0 \\
1+R_{\operatorname{mag}} \frac{k_{z}^{(\mathrm{i})}}{k_{z}^{(\mathrm{r})}} & =0 .
\end{aligned}
$$

The roundtrip gain in the resonator of Fig. 1 is then

$$
G=\frac{\left.k_{z}\right|_{v_{0}}}{\left.k_{z}\right|_{-v_{0}}},
$$

which is the same as Eq. (24). Therefore, the model developed here and the Dyakonov-Shur model give identical results for the roundtrip gain (and, hence, the instability increment) of a resonator comprising an electric and a magnetic boundary. The results will also coincide for any "hard" boundary with the surface impedance $Z_{\mathrm{s}}$, where the electric and magnetic fields are linked by the expression $E_{x}=Z_{\mathrm{s}} H_{y}$, see, e.g., Ref. 8.

However, the agreement between the two models cannot be expected to hold in general. In particular, the two models would disagree for the resonators comprising multiple channel sections with different electron concentrations. ${ }^{13,14}$ Ignoring the transverse field distributions might not be justified in general. In fully closed channels, the transverse electric fields of the counter-propagating plasmons differ below the channel, even though they almost coincide between the channel and the gate, see Fig. 3(a). In partially or fully open channels, the electric fields differ both above and below the channel, see Figs. 3(b) and 3(c). This difference and the need to satisfy the boundary condition everywhere along a boundary will influence the plasmonic reflection coefficient.

\section{CONCLUSIONS}

Using the mode-matching technique, this paper has considered amplification of $\mathrm{THz}$ plasmons in gated twodimensional channels. The plasmons amplify when they are incident upon an electric contact against the dc current, and they deamplify when incident along the dc current. The results have been obtained from electrodynamically rigorous boundary conditions and taking into account the complete field distributions. The amplification coefficient has been found both numerically and analytically; the analytical treatment has been further simplified for fully closed and open channels.

In symmetric resonators, comprising a homogeneous channel and identical boundaries, amplification at one contact is compensated by deamplification at the other. As a result, such resonators do not oscillate, in agreement with the traditional treatment by Dyakonov and Shur. ${ }^{1}$ To compare the two models further, this paper considered a resonator whose source is an electric conductor and the drain is a magnetic conductor. Then, the boundary conditions correspond to those postulated by Dyakonov and Shur. The two models give identical results.

However, this agreement can be expected to hold only for hard boundaries and not, for example, for resonators whose channels comprise sections with different electron concentrations. The latter are better candidates for asymmetric plasmonic oscillators, as realizing a magnetic boundary in practice would be a formidable challenge. Progress was made previously in analyzing such oscillators by the mode-matching technique. ${ }^{13,14}$ However, more work will be needed, using the present results, to describe amplification at the boundary between a gated and a non-gated channel.

\section{ACKNOWLEDGMENTS}

The author is grateful to Professor R. R. A. Syms and Professor V. V. Popov for helpful discussions.

\section{APPENDIX A: ANALYTICAL SOLUTION OF REFLECTION PROBLEM}

All the drifting quantities can be expanded in the Taylor series around $v_{0}=0$, and assuming the drift velocity is small, only the linear terms can be kept, so that

$$
\begin{aligned}
k_{z, x}^{(\mathrm{i}, \mathrm{r})} & \approx k_{z, x}^{(0)}+v_{0} \delta k_{z, x}^{(\mathrm{i}, \mathrm{r})} \\
R & \approx-1+v_{0} \delta R \\
r\left(k_{x}\right) & \approx 0+v_{0} \delta r(k x) \\
E_{x}^{(\mathrm{i}, \mathrm{r})} & \approx E_{x}^{(0)}+v_{0} \delta E_{x}^{(\mathrm{i}, \mathrm{r})} \\
e_{x}^{(\mathrm{r})} & \approx e_{x}^{(0)}+v_{0} \delta e_{x}^{(\mathrm{r})}
\end{aligned}
$$

Substituting these into the boundary condition Eq. (17) and keeping, again, only the terms linear in $v_{0}$ gives

$$
\delta E_{x}^{(\mathrm{i})}-\delta E_{x}^{(\mathrm{r})}+\delta R E_{x}^{(0)}+\int_{0}^{\infty} \delta r\left(k_{x}\right) e_{x}^{(0)} \mathrm{d} k_{x}=0 .
$$

Multiplying Eq. (A2) by $E_{x}^{(0)}$, integrating $\int_{-\infty}^{d} \mathrm{~d} x$, and using the property of mode orthogonality, gives

$$
\delta R=-\frac{\int_{-\infty}^{d}\left(\delta E_{x}^{(\mathrm{i})}-\delta E_{x}^{(\mathrm{r})}\right) E_{x}^{(0)} \mathrm{d} x}{\int_{-\infty}^{d} E_{x}^{(0) 2} \mathrm{~d} x} .
$$

The expressions for $E_{x}^{(0)}$ and $\delta E_{x}^{(\mathrm{i}, \mathrm{r})}$ can be found from Eq. (12) as

$$
\begin{aligned}
E_{x}^{(0)}= & \frac{\cos \left[k_{x}^{(0)}(x-d)\right]}{\sin \left(k_{x}^{(0)} d\right)} \\
\delta E_{x}^{(\mathrm{i}, \mathrm{r})}= & -\frac{\delta k_{x}^{(\mathrm{i}, \mathrm{r})}}{\sin \left(k_{x}^{(0)} d\right)}\left[(x-d) \sin \left[k_{x}^{(0)}(x-d)\right]\right. \\
& \left.+d \cot \left(k_{x}^{(0)} d\right) \cos \left[k_{x}^{(0)}(x-d)\right]\right]
\end{aligned}
$$


above the channel and

$$
\begin{aligned}
E_{x}^{(0)} & =\mathrm{j} \exp \left(-\mathrm{j} k_{x}^{(0)} x\right) \\
\delta E_{x}^{(\mathrm{i}, \mathrm{r})} & =\delta k_{x}^{(\mathrm{i}, \mathrm{r})} x \exp \left(-\mathrm{j} k_{x}^{(0)} x\right)
\end{aligned}
$$

below the channel. Substituting Eqs. (A4) and (A5) into Eq. (A3) gives Eq. (18). Similarly, expanding the dispersion relation Eq. (9) into a Taylor series and keeping only the terms linear in $v_{0}$ gives Eq. (18).

\section{APPENDIX B: NUMERICAL SOLUTION OF REFLECTION PROBLEM}

The reflection problem Eq. (17) can be solved numerically by the following method. Multiplying Eq. (17) by $E_{x}^{(\mathrm{r})}$ and integrating $\int_{-\infty}^{d} \mathrm{~d} x$ gives

$$
\begin{aligned}
& k_{x}^{(\mathrm{r})} \frac{\Gamma^{(\mathrm{r})} k_{x}^{(\mathrm{i})}-\Gamma^{(\mathrm{i})} k_{x}^{(\mathrm{r})}}{k_{x}^{(\mathrm{i}) 2}-k_{x}^{(\mathrm{r}) 2}}+R\left(\mathrm{j} \frac{k_{x}^{(\mathrm{r})} d}{4 \sin ^{2}\left(k_{x}^{(\mathrm{r})} d\right)}+\frac{\Gamma^{(\mathrm{r})}}{2}\right) \\
& +\int_{0}^{\infty} r\left(k_{x}\right) k_{x}^{(\mathrm{r})} \frac{\Gamma k_{x}^{(\mathrm{r})}-\Gamma^{(\mathrm{r})} k_{x}}{k_{x}^{(\mathrm{r}) 2}-k_{x}^{2}}=0 .
\end{aligned}
$$

Likewise, multiplying Eq. (17) by $e_{x}^{(\mathrm{r})}\left(\tilde{k}_{x}\right)$ and integrating gives

$$
\begin{aligned}
& \frac{\Gamma^{(\mathrm{i})} \tilde{k}_{x}-\tilde{\Gamma} k_{x}^{(\mathrm{i})}}{k_{x}^{(\mathrm{i})} 2-\tilde{k}_{x}^{2}}+R \frac{\Gamma^{(\mathrm{r})} \tilde{k}_{x}}{k_{x}^{(\mathrm{r})} 2-\tilde{k}_{x}^{2}}+\pi r\left(\tilde{k}_{x}\right) \\
& \quad \times\left(\frac{1}{4 \mathrm{j} \sin ^{2}\left(\tilde{k}_{x} d\right)}+\mathrm{j} \tilde{\Gamma}^{2}+\tilde{\Gamma} \cot \left(\tilde{k}_{x} d\right)\right) \\
& +f_{0}^{\infty} r\left(k_{x}\right) \frac{\tilde{\Gamma} k_{x}-\Gamma \tilde{k}_{x}}{\tilde{k}_{x}^{2}-k_{x}^{2}} \mathrm{~d} k_{x}=0,
\end{aligned}
$$

where $f$ denotes Cauchy principal value. Equations (B1) and (B2) can be solved numerically, for example, by discretizing the integrals and presenting the equations in a matrix form.

${ }^{1}$ M. Dyakonov and M. Shur, Phys. Rev. Lett. 71, 2465 (1993).

${ }^{2}$ M. Dyakonov and M. Shur, IEEE Trans. Electron. Devices 43, 380 (1996).

${ }^{3}$ J. B. Gunn, Phys. Lett. 4, 194 (1963).

${ }^{4}$ L. Solymar and E. A. Ash, Int. J. Electron. 20, 127 (1966).

${ }^{5}$ M. C. Steel and B. Vural, Wave Interactions in Solid State Plasmas (McGraw-Hill, New York, 1969).

${ }^{6}$ J. R. Pierce, J. Appl. Phys. 15, 721 (1944).

${ }^{7}$ F. J. Crowne, J. Appl. Phys. 82, 1242 (1997).

${ }^{8}$ M. V. Cheremisin and G. G. Samsonidze, Semiconductors 33, 578 (1999).

${ }^{9}$ F. J. Crowne, J. Appl. Phys. 87, 8056 (2000).

${ }^{10}$ M. Dyakonov and M. S. Shur, Appl. Phys. Lett. 87, 111501 (2005).

${ }^{11}$ O. Sydoruk, R. R. A. Syms, and L. Solymar, Appl. Phys. Lett. 97, 263504 (2010).

${ }^{12}$ V. V. Popov, G. M. Tsymbalov, and M. S. Shur, J. Phys.: Condens. Matter 20, 384208 (2008).

${ }^{13}$ O. Sydoruk, R. R. A. Syms, and L. Solymar, Opt. Express 20, 19618 (2012).

${ }^{14}$ O. Sydoruk, R. R. A. Syms, and L. Solymar, J. Appl. Phys. 112, 104512 (2012).

${ }^{15}$ O. Sydoruk, J. Phys. D: Appl. Phys. 46, 135103 (2013).

${ }^{16}$ O. Sydoruk, J. Phys. D: Appl. Phys. 46, 345101 (2013).

${ }^{17}$ V. V. Shevchenko, Continuous Transitions in Open Waveguides (Golem Press, Boulder, Colorado, 1971)

${ }^{18}$ D. Marcuse, Light Transmission Optics (Van Nostrand Reinhold, New York, 1982).

${ }^{19}$ T. N. Theis, Surf. Sci. 98, 515 (1980).

${ }^{20}$ D. C. Tsui, E. Gornik, and R. A. Logan, Solid State Commun. 35, 875 (1980).

${ }^{21}$ A. L. Fetter, Ann. Phys. 88, 1 (1974).

${ }^{22}$ A. D. Bresler, G. H. Joshi, and N. Marcuvitz, J. Appl. Phys. 29, 794 (1958).

${ }^{23}$ R. E. Collin, Foundations for Microwave Engineering (Wiley-IEEE Press, Hoboken, NJ, 2001). 